\title{
On certain differential identities in prime rings with involution
}

Mohammad Ashraf and Mohammad Aslam Siddeeque 


\title{
ON CERTAIN DIFFERENTIAL IDENTITIES IN PRIME RINGS WITH INVOLUTION
}

\author{
MOHAMMAD ASHRAF AND MOHAMMAD ASLAM SIDDEEQUE \\ Received 27 December, 2013
}

\begin{abstract}
In the present paper we investigate commutativity of $*$-prime ring $R$, which satisfies certain differential identities on $*$-ideals of $R$. Some results already known for prime rings on ideals have also been deduced. Finally, we provide several examples to justify that various restrictions imposed in the hypotheses of our theorems are not superfluous.
\end{abstract}

2010 Mathematics Subject Classification: 16W25; 16N60; 16U80; 16W10

Keywords: derivation, $*$-prime ring, $*$-ideal and commutativity

\section{INTRODUCTION}

Throughout the paper, $R$ will represent an associative ring with center $Z$. For any $x, y \in R$ the symbol $[x, y]$ will denote the commutator $x y-y x$; while the symbol $x o y$ will stand for the anticommutator $x y+y x$. $R$ is called a prime ring if $x R y=\{0\}$ implies $x=0$ or $y=0$. It is called semiprime if $x R x=\{0\}$ implies $x=0$. Given an integer $n>1$, ring $R$ is said to be $n$-torsion free, if for $x \in R, n x=0$ implies $x=0$. An additive mapping $x \mapsto x^{*}$ of $R$ into itself is called an involution on $R$ if it satisfies the conditions: $(i)\left(x^{*}\right)^{*}=x$, $(i i)(x y)^{*}=y^{*} x^{*}$ for all $x, y \in R$. A ring $R$ equipped with an involution ' $*$ ' is called a ring with involution or a $*$-ring. A ring $R$ with involution ' $*$ ' is said to be $*$-prime if $a R b=a R b^{*}=\{0\}$, where $a, b \in R$ ( equivalently $a R b=a^{*} R b=\{0\}$ ) implies that either $a=0$ or $b=0$. It is to be noted that every prime ring having an involution ' $*$ ' is $*$-prime but the converse is not true in general. Of course, if $R^{o}$ denotes the opposite ring of a prime ring $R$, then $R \times R^{o}$ equipped with the exchange involution $*_{e x}$, defined by $*_{e x}(x, y)=(y, x)$, is $*_{e x}$-prime but not prime. Let $R$ be a $*$-prime ring. The set of symmetric and skew symmetric elements of $R$ will be denoted by $S a_{*}(R)$ i.e.; $S a_{*}(R)=\left\{x \in R \mid x^{*}= \pm x\right\}$. An ideal $I$ of $R$ is called a $*$-ideal of $R$ if $I^{*}=I$. An additive mapping $d: R \longrightarrow R$ is said to be a derivation on $R$ if $d(x y)=d(x) y+x d(y)$ holds for all $x, y \in R$. Let $R$ be a $*$-prime ring, $a \in R$ and $a R a=\{0\}$. This implies that $a R a R a^{*}=\{0\}$ also. Now $*$-primeness of $R$ insures that $a=0$ or $a R a^{*}=\{0\} . a R a^{*}=\{0\}$ together with $a R a=\{0\}$ gives us $a=0$. Thus we conclude that every $*$-prime ring is a semiprime 
ring.

Ashraf, Bell, Herstein and Hongon etc. [1-4] studied the commutativity of prime and semiprime rings, satisfying certain differential identities on some appropriate subsets of $R$. Hence it is natural to question that what can we say about the commutativity of $*$-prime rings in which derivations satisfy certain identities on $*$-ideals. In this direction Oukhtite, Salhi, Vukman and Kosi-Ulbl etc. [5-11] have already investigated several differential identities on $*$-ideals. In this paper we have obtained the commutativity of $*$-prime rings satisfying any one of the following differential identities on $*$-ideal $I$ under some restrictions: $(i) d($ xoy $)=d(x)$ oy $(i \mathrm{i})$ $d(x)$ oy $=$ xoy,, (iii) $d([x, y])= \pm(x o y)$ (iv) $d(x o y)= \pm[x, y],(v) d(x)$ oy $\in Z$, (vi) $d([x, y]) \pm($ xoy $) \in Z$, (vii $) d(x o y) \pm[x, y] \in Z$, (vii i $) d(x) o d(y)=x o y$ and (ix) $(d(x)$ oy $)-(\operatorname{xod}(y)) \in Z$ for all $x, y \in I$. We have also shown that there exists no nonzero derivation $d$ satisfying the following differential identities on $*$-ideal $I$ in a $*$-prime ring $R$ under some constraints viz.; $(i) d(x o y)=d(x)$ oy $(i i) d(x)$ oy $=$ $x o y,($ i i i $) d(x)$ oy $=x o d(y),($ iv $) d(x) o y=d(x) \operatorname{od}(y)(v) \operatorname{xod}(y)=d(x) \operatorname{od}(y)$ for all $x, y \in I$. Several examples have also been constructed to justify the necessity of $*$-primeness or "characteristic different from 2 " of the ring $R$ while dealing with the above differential identities.

\section{PReliminary Results}

We begin with the following lemmas which are essential for developing the proof of our main results. The proofs of Lemma 1-3 can be seen in [6] while that of Lemma 4 can be found in [8].

Lemma 1. Let $R$ be $a *$-prime ring and $I$ be a nonzero $*$-ideal of $R$. If $x, y \in R$ satisfy $x I y=x I y^{*}=\{0\}$, then $x=0$ or $y=0$.

Lemma 2. Let $R$ be $a *$-prime ring admitting a nonzero derivation $d$ which commutes with ' $*$ '. If I is a nonzero $*$-ideal of $R$ and $[x, R] \operatorname{Id}(x)=\{0\}$ for all $x \in I$, then $R$ is commutative.

Lemma 3. Let $R$ be a $*$-prime ring admitting a nonzero derivation $d$ which commutes with ' $*$ '. If $I$ is a nonzero $*$-ideal of $R$ and $[d(x), x]=0$ for all $x \in I$, then $R$ is commutative.

Lemma 4. Let $d$ be a nonzero derivation of a 2-torsion free $*$-prime ring $R$ and $I$ a nonzero $*$-ideal of $R$. If $r \in S a_{*}(R)$ satisfies $[d(x), r]=0$ for all $x \in I$, then $r \in Z$. Furthermore, if $d(I) \subseteq Z$, then $R$ is commutative.

Now we prove the following:

Lemma 5. If $R$ is a $*$-prime ring admitting a nonzero central $*$-ideal $I$ i.e.; $I \subseteq$ $Z$, then $R$ is commutative. 
Proof. Let $r, s \in R$ and $x \in I$. Using hypothesis we get $r s x=r x s=s r x$. This implies that $[r, s] I=\{0\}$ and hence $[r, s] I l=[r, s] I l^{*}=\{0\}$, where $0 \neq l \in R$. In view of Lemma 1 , we get the required result.

Lemma 6. Let $R$ be $a *$-prime ring admitting a nonzero derivation $d$ which commutes with ' $*$ '. If $I$ is a nonzero $*$-ideal of $R$ and $d(x) I[x, R]=\{0\}$ for all $x \in I$, then $R$ is commutative.

Proof. For the proof, first we show that $d(x) I[x, R]=\{0\}$ for all $x \in I$ if and only if $[x, R] I d(x)=\{0\}$ for all $x \in I$. Suppose that $d(x) I[x, R]=\{0\}$ for all $x \in I$. This implies that $[x, R]^{*} I^{*}\{d(x)\}^{*}=\{0\}$ for all $x \in I$. Since $I$ is a $*$-ideal of $R$ and $d$ commutes with ' $*$ ', we conclude that $\left[x^{*}, R\right] \operatorname{Id}\left(x^{*}\right)=\{0\}$ for all $x \in I$. Now replacing $x$ by $x^{*}$ in the last relation we obtain that $[x, R] I d(x)=\{0\}$ for all $x \in I$. Converse can be proved in similar way. Finally using Lemma 2 , we get the required result.

Lemma 7. If $R$ is a *-prime ring of characteristic different from 2, then $R$ is 2-torsion free.

Proof. Suppose that $x \in R$ such that $2 x=0$. This implies that $2 x r s=0$ for all $r, s \in R$ i.e.; $x R(2 s)=\{0\}$ for all $s \in R$. Since characteristic of $R$ is different from 2 and $R \neq\{0\}$, this provides us a nonzero element $l \in R$ such that $2 l \neq 0$. Now we conclude that $x R(2 l)=\{0\}=x R(2 l)^{*}$. Finally $*$-primeness of $R$ provides us $x=0$ and hence $R$ is 2 -torsion free.

\section{MAIN RESULTS}

There has been a great deal of work concerning commutativity of prime and semiprime rings satisfying certain differential identities on some appropriate subsets of the ring, see [1-4] where further references can be found. Motivated by such work, we explore the commutativity of $*$-prime ring involving derivations satisfying some identities on nonzero $*$-ideal of the ring. We begin with the following differential identity on a nonzero $*$-ideal of a $*$-prime ring, which is of independent interest and insures the commutativity of the ring:

Theorem 1. Let $R$ be a $*$-prime ring, $I$ be a nonzero $*$-ideal of $R$ and $d$ a nonzero derivation of $R$ such that $d$ commutes with ' $*$ '. If $d(x \circ y)=d(x)$ oy for all $x, y \in I$ or $d(x)$ oy $=x$ oy for all $x, y \in I$, then $R$ is commutative.

Proof. Assume that $d(x o y)=d(x)$ oy for all $x, y \in I$. Now replacing $y$ by $x y$ we arrive at $d(x \circ x y)=d(x) o x y$ i.e.; $d(x)(x o y)+x d(x o y)=d(x) x y+x y d(x)$. Using hypothesis we obtain that $d(x)(x o y)+x(d(x)$ oy $)=d(x) x y+x y d(x)$. This implies that $d(x) x y+d(x) y x+x d(x) y+x y d(x)=d(x) x y+x y d(x)$ i.e.;

$$
d(x) y x=-x d(x) y
$$


for all $x, y \in I$. Putting $y r$, where $r \in R$, for $y$ in the relation (3.1) and using it again we conclude that $d(x) y r x=-x d(x) y r=d(x) y x r$ i.e.; $d(x) I[x, R]=\{0\}$ for all $x \in I$ and Lemma 6 forces that $R$ is commutative.

Now suppose that $d(x)$ oy $=x$ oy for all $x, y \in I$. Replacing $x$ by $y x$, we get $d(y x) o y=y x o y$ i.e.; $d(y x) o y=y(x o y)$ for all $x, y \in I$. Using our hypothesis we obtain that $d(y x) y+y d(y x)=y(d(x) o y)$ i.e.; $d(y) x y+y d(x) y+y d(y) x+$ $y^{2} d(x)=y d(x) y+y^{2} d(x)$ and therefore $d(y) x y=-y d(y) x$ for all $x, y \in I$. In view of the latter relation we arrive at $d(x) y x=-x d(x) y$ for all $x, y \in I$. This is identical with the relation (3.1). Arguing as in above we conclude that $R$ is commutative.

Theorem 2. Let $R$ be a *-prime ring of characteristic different from $2, I$ be a nonzero $*$-ideal of $R$ and $d$ a derivation of $R$ such that ' $d$ ' commutes with ' $*$ '. If $d(x o y)=d(x)$ oy for all $x, y \in I$ or $d(x)$ oy $=x$ oy for all $x, y \in I$, then $d=0$

Proof. Suppose that $d(x o y)=d(x)$ oy for all $x, y \in I$. Then we have to show that $d=0$. Suppose on contrary that $d \neq 0$. Therefore by Theorem 1 we conclude that $R$ is commutative. By hypothesis given we have $2 d(x y)=2 d(x) y$ for all $x, y \in I$ and hence $d(x) y+x d(y)=d(x) y$ for all $x, y \in I$. This yields that $x d(y)=0$ for all $x, y \in I$ and since $R$ is commutative we arrive at $d(x) y=0$ for all $x, y \in I$. Replacing $y$ by $s y$ where $s \in R$ in the last relation we obtain that $d(x) s y=0$ i.e.; $d(x) R y=\{0\}$ for all $x, y \in I$. Since $I$ is a $*$-ideal of $R$, we conclude that $d(x) R y^{*}=\{0\}$ for all $x, y \in I$ also. $I \neq\{0\}$ and $*$-primeness of $R$ provide us $d(x)=0$ for all $x \in I$. Now putting $x t$ where $t \in R$ in place of $x$ in the last relation and using the same again we arrive at $x d(t)=0$ i.e.; $I d(t)=\{0\}$. Using hypothesis this relation provides us $l I d(t)=l I\{d(t)\}^{*}=\{0\}$ where $0 \neq l \in R$. Finally Lemma 1 assures that $d=0$, leading to a contradiction.

Now assume that $d(x)$ oy $=x$ oy for all $x, y \in I$. Then we have to show that $d=0$. If $d \neq 0$, then by Theorem 1 we conclude that $R$ is commutative. By hypothesis given we have $2 d(x) y=2 x y$ for all $x, y \in I$, then $d(x) y=x y$ for all $x, y \in I$. Replacing $x$ by $r x$, where $r \in R$ in the last relation and using the same again we infer that $d(r x) y=r x y$ i.e.; $d(r) x y+r d(x) y=r x y$. This implies that $d(r) x y=0$ for all $r \in R$ and $x, y \in I$. Finally we conclude that $d(r) I y=\{0\}$ for all $r \in R$ and $y \in I$. In particular we also obtain that $d(r) I y^{*}=\{0\}$. Lemma 1 and $I \neq\{0\}$ assure that $d=0$, leading to a contradiction.

The following example shows that the existence of "characteristic different from 2 " in the hypothesis of the above theorem is not superfluous.

Example 1. Suppose that $R=\mathbb{Z}_{2}[x] \times \mathbb{Z}_{2}[x]$, where $\mathbb{Z}_{2}[x]$ is the polynomial ring over $\mathbb{Z}_{2}$. Let us consider $D, *: R \longrightarrow R$ such that

$$
D(f(x), g(x))=(d(f(x)), d(g(x))) \text { and }(f(x), g(x))^{*}=(g(x), f(x)),
$$


where $d$ is the usual differentiation in $\mathbb{Z}_{2}[x]$. It is easy to check that $R$ is a $*$ ex -prime ring since ' $*$ ' is an involution of $R$, known as exchange involution denoted by $*_{e x}$ and $D$ is a derivation on $R$. Moreover, it is obvious that $R$ is a ring of characteristic 2 and $D *_{e x}=*_{e x} D$. Further assume that $I=\left[x^{2}\right]$ is the ideal of $\mathbb{Z}_{2}[x]$ generated by $x^{2} \in \mathbb{Z}_{2}[x]$. Then it can be easily shown that $d=I \times I$ is a nonzero $*_{e x}$-ideal of $R$ such that $D(x o y)=D(x)$ oy for all $x, y \in \mathcal{d}$ and $D(x) o y=x o y$ for all $x, y \in \mathcal{d}$. However $D \neq 0$.

In the year 2007, Oukhtite and Salhi [9] obtained the commutativity of $*$-prime ring $R$ having "characteristic different from 2" and admitting a nonzero derivation $d$ which commutes with ' $*$ ', such that $d([x, y])=0$ for all $x, y$ in a nonzero $*$-ideal of $R$. We have improved this result and showed that the restriction of "characteristic different from 2" on $R$ used in the above theorem is redundant. In addition we have also investigated similar other differential identities which insure the commutativity of $*$-prime rings. In fact we have obtained the following.

Theorem 3. Let $R$ be a $*$-prime ring and I a nonzero $*$-ideal of $R$. If $R$ admits a nonzero derivation $d$ which commutes with ' $*$ ' and satisfies any one of the following differential identities: $(i) d([x, y])=0$ for all $x, y \in I,(i i) d([x, y])= \pm[x, y]$ for all $x, y \in I$, (iii $) d([x, y])= \pm(x o y)$ for all $x, y \in I,(i v) d(x o y)=0$ for all $x, y \in I,(v) d(x o y)= \pm(x o y)$ for all $x, y \in I$ and $(v i) d(x o y)= \pm[x, y]$ for all $x, y \in I$. Then $R$ is commutative.

Proof. (i) By hypothesis we have $d([x, y])=0$, for all $x, y \in I$. Now replacing $y$ by $y x$ and using the hypothesis, we obtain that $[x, y] d(x)=0$ for all $x, y \in I$ i.e.;

$$
x y d(x)=y x d(x)
$$

for all $x, y \in I$. Replacing $y$ by $r y$, where $r \in R$ in the relation (3.2) and using it again, we arrive at $[x, r] y d(x)=0$ for all $x, y \in I$ and for all $r \in R$ i.e.; $[x, R] \operatorname{Id}(x)=\{0\}$ for all $x \in I$. Now by Lemma 2 , the result follows.

(ii ) By hypothesis we have $d([x, y])= \pm[x, y]$, for all $x, y \in I$. Now replacing $y$ by $y x$ and using the hypothesis, we infer that $[x, y] d(x)=0$ for all $x, y \in I$ i.e.; $x y d(x)=y x d(x)$ for all $x, y \in I$. This is identical with the relation (3.2). Now arguing in the similar way as above $(i)$, we get our required result.

(i i i ) Using the same trick as used in ( $i$ i ), result follows.

(iv) By hypothesis we have $d(x o y)=0$, for all $x, y \in I$. Now replacing $y$ by $y x$ and using the hypothesis, we obtain that $(x o y) d(x)=0$ for all $x, y \in I$ i.e.;

$$
x y d(x)=-y x d(x)
$$

for all $x, y \in I$. Replacing $y$ by $r y$, where $r \in R$ in the relation (3.3) and using it again, we arrive at $[x, r] y d(x)=0$ for all $x, y \in I$ and for all $r \in R$ i.e.; 
$[x, R] \operatorname{Id}(x)=\{0\}$ for all $x \in I$. Now by Lemma 2, the result follows.

(v) By hypothesis we have $d(x o y)= \pm(x o y)$, for all $x, y \in I$. Now replacing $y$ by $y x$ and using the hypothesis, we conclude that $(x o y) d(x)=0$ for all $x, y \in I$ i.e.; $x y d(x)=-y x d(x)$ for all $x, y \in I$. This is identical with the relation (3.3). Now using similar arguments as used in (iv), we get our required result.

(vi) Using the same arguments as used in (v), result follows.

Corollary 1. Let $R$ be a prime ring and $I$ a nonzero ideal of $R$. If $R$ admits a nonzero derivation $d$ satisfying any one of the following differential identities: (i) $d([x, y])=0$ for all $x, y \in I$, (ii $) d([x, y])= \pm[x, y]$ for all $x, y \in I$, (iii) $d([x, y])= \pm$ (xoy) for all $x, y \in I$, (iv) $d(x o y)=0$ for all $x, y \in I,(v)$ $d(x \circ y)= \pm(x o y)$ for all $x, y \in I$ and $(v i) d(x o y)= \pm[x, y]$ for all $x, y \in I$. Then $R$ is commutative.

Proof. Let $d$ be a nonzero derivation of $R$ satisfying any one of above differential identities. Since $R$ is a prime ring, consider $\mathcal{R}=R \times R^{o}$, which is clearly a $*_{e x}$ prime ring. Set $d=I \times I^{o}$ is a nonzero $*_{e x}$-ideal of $\mathcal{R}$. Now define $D: \mathcal{R} \longrightarrow \mathcal{R}$ by $D(x, y)=(d(x), d(y))$. Using hypothesis it can be easily proved that $D$ is a nonzero derivation of $\mathcal{R}$. Moreover $D *_{e x}=*_{e x} D$ and $(i) D([x, y])=0$ for all $x, y \in \mathcal{d}$, (ii $) D([x, y])= \pm[x, y]$ for all $x, y \in d$ (iii $) D([x, y])= \pm(x o y)$ for all $x, y \in \mathcal{R},($ iv $) D(x o y)=0$ for all $x, y \in \mathcal{d},(v) D(x o y)= \pm(x o y)$ for all $x, y \in \mathcal{d}$ and (vi) $D(x o y)= \pm[x, y]$ for all $x, y \in \mathcal{\ell}$. Using the Theorem 3, we deduce that $\mathcal{R}$ is commutative and in turn we obtain that $R$ is also commutative.

Theorem 4. Let $R$ be a *-prime ring of characteristic different from 2 and $I$ a nonzero $*$-ideal of $R$. If $R$ admits a nonzero derivation $d$ such that $d(x)$ oy $\in Z$ for all $x, y \in I$, then $R$ is commutative.

Proof. Assume that

$$
d(x) \text { oy } \in Z
$$

for all $x, y \in I$. The relation (3.4) implies that $d(x) y+y d(x) \in Z$ for all $x, y \in I$. Since $I$ is a nonzero ideal of $R, d(x) y+y d(x) \in I$ for all $x, y \in I$ also. Now we conclude that $d(x) y+y d(x) \in Z \cap I$ for all $x, y \in I$. Now we break the proof in two cases.

Case I: If $Z \cap I=\{0\}$, we obtain that $d(x) y+y d(x)=0$ i.e.;

$$
d(x) y=-y d(x)
$$

for all $x, y \in I$. Substituting $r y$, where $r \in R$ for $y$ in the relation (3.5) and using it again we arrive at $d(x) r y=r d(x) y$ i.e.; $[d(x), R] y=\{0\}$. This implies that $[d(x), R] I s=\{0\}=[d(x), R] I s^{*}$, where $0 \neq s \in R$. Now by Lemma 1 , we infer that $[d(x), R]=\{0\}$ for all $x \in I$ i.e; $d(I) \subseteq Z$. Finally, Lemma 4 assures that $R$ is commutative. 
Case II: If $Z \cap I \neq\{0\}$, there exists $0 \neq z \in Z \cap I$. By hypothesis we have $d(x) y+$ $y d(x) \in Z$ for all $x, y \in I$. In particular we conclude that $d(x) z+z d(x) \in Z$ i.e.; $2 d(x) z \in Z$ for all $x \in I$. Now we have $2 d(x) z r=2 r d(x) z$ for all $x \in I$ and $r \in R$. This yields that $[d(x), r] z=0$ for all $x \in I$ and $r \in R$ i.e.; $[d(x), R] I z=\{0\}$ for all $x \in I$. We already know that $0 \neq z \in Z \cap I$. Since $I=I^{*}$ and $Z=Z^{*}$, the latter relation implies that $0 \neq z^{*} \in Z \cap I$. Now using $z^{*}$ in place of $z$ and arguing in the similar way as in just above lines we arrive at $[d(x), R] I z^{*}=\{0\}$ for all $x \in I$. Finally we conclude that $[d(x), R] I z=\{0\}=[d(x), R] I z^{*}$ for all $x \in I$, where $0 \neq z$. Using Lemma $1 \&$ Lemma 4, we get the required result for this case.

The following example demonstrates that the $*$-primeness in the hypothesis of the above theorem can not be omitted.

Example 2. Let $R=\mathbb{R}[x] \times \mathbb{H}$, where $\mathbb{R}[x]$ is the polynomial ring over the ring $\mathbb{R}$ of real numbers and $\mathbb{E}$ is the ring of real quaternions. $R$ is clearly a ring of characteristic different from 2. Define $D: R \longrightarrow R$ as $D(f(x), q)=(d(f(x), 0)$, where $d$ is the usual differentiation of the polynomial ring $\mathbb{R}[x]$. Also define $*: R \rightarrow R$ as $*(f(x), q)=(f(-x), \bar{q})$, where $f(x) \in \mathbb{R}[x]$ and $\bar{q}=\alpha-\beta i-\gamma j-\delta k$, where $q=\alpha+\beta i+\gamma j+\delta k \in \mathbb{W}$. It can be easily shown that $D$ and ' $*$ ' are a nonzero derivation and an involution of $R$ respectively. Suppose that $I=\mathbb{R}[x] \times\{0\}$. It is obvious that $I$ is a $*$-ideal of $R$. Let $0 \neq u(x) \in \mathbb{R}[x]$ and $0 \neq v \in \mathbb{H}$. Then we have $(u(x), 0) R(0, v)=\{(0,0)\}=(u(x), 0) R(0, v)^{*}$, where $(0,0) \neq(u(x), 0),(0,0) \neq$ $(0, v) \in R$. This implies that $R$ is not a $*$-prime ring but it is a semiprime ring. It can be easily seen that $D(m) o n \in Z$ for all $m, n \in I$ but $R$ is noncommutative.

Theorem 5. Let $R$ be a $*$-prime ring of characteristic different from 2 , I a nonzero $*$-ideal of $R$. If $R$ admits a nonzero derivation d which commutes with '*' such that $d([x, y]) \pm(x \circ y) \in Z$ for all $x, y \in I$. Then $R$ is commutative.

Proof. It is clear that $d([x, y]) \pm(x o y) \in I$ for all $x, y \in I$ also. Now in view of our hypothesis we conclude that $d([x, y]) \pm(x o y) \in Z \cap I$ for all $x, y \in I$.

Case I: If $Z \cap I=\{0\}$, then $d([x, y]) \pm(x o y)=0$ for all $x, y \in I$, using Theorem 3 we get our required result.

Case II: If $Z \cap I \neq\{0\}$, then suppose $0 \neq z \in Z \cap I$. Replacing $y$ by $z$, we arrive at $d([x, z]) \pm(x o z) \in Z \cap I$ for all $x \in I$ i.e.; $2 x z \in Z$ for all $x \in I$ and hence $x z \in Z$ for all $x \in I$ i.e.; $x z r=r x z$ for all $r \in R$. This implies that $[x, R] R z=\{0\}$ for all $x \in I$. Since $0 \neq z^{*} \in Z \cap I$, arguing in the similar lines as above we also obtain that $[x, R] R z^{*}=\{0\}$ for all $x \in I$. By $*$-primeness of $R$ we conclude that $I \subseteq Z$. Finally by Lemma 5 , the result follows.

Theorem 6. Let $R$ be a $*$-prime ring of characteristic different from 2 and $I$ a nonzero $*$-ideal of $R$. If $R$ admits a nonzero derivation $d$ which commutes with '*' such that $d(x o y) \pm[x, y] \in Z$ for all $x, y \in I$. Then $R$ is commutative. 
Proof. It is clear that $d(x o y) \pm[x, y] \in I$ for all $x, y \in I$ also. Now including the hypothesis we conclude that $d(x o y) \pm[x, y] \in Z \cap I$ for all $x, y \in I$.

Case I: If $Z \cap I=\{0\}$, we find that $d(x o y) \pm[x, y]=0$ for all $x, y \in I$ and hence using Theorem 3 we get our required result.

Case II: Suppose $Z \cap I \neq\{0\}$. Let $0 \neq z \in Z \cap I$. Replacing $y$ by $z$, we arrive at $d(x o z) \in Z \cap I$ for all $x \in I$ i.e.; $2 d(x z) \in Z$ for all $x \in I$ and hence $d(x z) \in Z$ for all $x \in I$ i.e.; $d(x) z x+x d(z) x=x d(x) z+x x d(z)$ for all $x \in I$. Using the fact that $d(Z) \subseteq Z$, we conclude that $[d(x), x] R z=\{0\}$ for all $x \in I$. since $Z^{*}=Z$ and $I^{*}=I$, we obtain that $0 \neq z^{*} \in Z \cap I$. Now arguing in the similar lines as above we also obtain that $[d(x), x] R z^{*}=\{0\}$ for all $x \in I$. By $*$-primeness of $R$ we conclude that $[d(x), x]=0$ for all $x \in I$. Finally by Lemma 3, the result follows.

Corollary 2. Let $R$ be a prime ring of characteristic not $2, I$ a nonzero ideal and $d$ a nonzero derivation of $R$ satisfying either of the following differential identities (i) $d([x, y]) \pm(x o y) \in Z$ for all $x, y \in I$ or $(i i) d(x o y) \pm[x, y] \in Z$ for all $x, y \in I$. Then $R$ is commutative.

Proof. Assume that $d$ is a nonzero derivation of $R$ such that $(i) d([x, y]) \pm(x o y) \in$ $Z$ for all $x, y \in I$ or $(i i) d(x o y) \pm[x, y] \in Z$ for all $x, y \in I$. Since $R$ is a prime ring of characteristic not 2 , consider $\mathcal{R}=R \times R^{o}$, which is clearly a $*_{\text {ex }}$-prime ring of characteristic different from 2. Set $d=I \times I^{o}$ a nonzero $*_{e x}$-ideal of $\mathcal{R}$. Now define $D: \mathcal{R} \longrightarrow \mathcal{R}$ by $D(x, y)=(d(x), d(y))$. Using hypothesis it can be easily proved that $D$ is a nonzero derivation of $\mathcal{R}$. Moreover $D *_{e x}=*_{e x} D$ and $(i)$ $D([x, y]) \pm(x o y) \in Z(\mathcal{R})$ for all $x, y \in \mathcal{l}$ or $(i i) D(x o y) \pm[x, y] \in Z(\mathcal{R})$ for all $x, y \in \mathcal{l}$. In view of the Theorem 5 and Theorem 6 we deduce that $\mathcal{R}$ is commutative and in turn we obtain that $R$ is also commutative.

The following example shows that the $*$-primeness in the hypotheses of the Theorems $5 \& 6$ can not be omitted.

Example 3. Let $R=\mathbb{R}[x] \times \mathbb{H}$, where $\mathbb{R}[x]$ is the polynomial ring over ring $\mathbb{R}$ of real numbers and $\mathbb{t}$ is the ring of real quaternions. Clearly, $R$ is a ring of characteristic different from 2. Define $D: R \longrightarrow R$ as $D(f(x), q)=\left(0, d_{i}(q)\right)$, where $d_{i}$ is the inner derivation of $\mathbb{R}$, determined by $i \in \mathbb{W}$, i.e.; $d_{i}(q)=[i, q]$ for all $q \in \mathbb{W}$. Also define $*: R \longrightarrow R$ as $*(f(x), q)=(f(x), \bar{q})$, where $f(x) \in \mathbb{R}[x]$ and $\bar{q}=$ $\alpha-\beta i-\gamma j-\delta k$, where $q=\alpha+\beta i+\gamma j+\delta k \in \mathbb{W}$. It can be easily shown that $D$ and $*$ are a nonzero derivation and an involution of $R$ respectively such that $D *=* D$. Suppose that $I=\mathbb{R}[x] \times\{0\}$. It is obvious that $I$ is a $*$-ideal of $R$. Let $0 \neq u(x) \in \mathbb{R}[x]$ and $0 \neq v \in \mathbb{W}$. Then we have $(u(x), 0) R(0, v)=\{(0,0)\}=(u(x), 0) R(0, v)^{*}$, where $(0,0) \neq(u(x), 0),(0,0) \neq(0, v) \in R$. This implies that $R$ is not a $*$-prime ring but it is a semiprime ring. Here it is obvious to observe that $(i) D([m, n]) \pm(m o n) \in Z$ for all $m, n \in I$ and (ii) $D(m o n) \pm[m, n] \in Z$ for all $m, n \in I$, but $R$ is noncommutative. 
We now consider differential identities involving anticommutators in the next two results and show that there does not exist nonzero derivation satisfying these differential identities.

Theorem 7. Let $R$ be $a *$-prime ring of characteristic different from 2 and $I$ a nonzero $*$-ideal of $R$ such that $Z \cap I \neq\{0\}$. Then there exists no nonzero derivation $d$ such that $d(x)$ oy $=x o d(y)$ for all $x, y \in I$.

Proof. By hypothesis we have $d(x) y+y d(x)-x d(y)-d(y) x=0$ for all $x, y \in$ $I$. Let $z \in Z \cap I$. Replacing $y$ by $z$ in the hypothesis, we arrive at $d(x) z+z d(x)-$ $x d(z)-d(z) x=0$ for all $x \in I$ and for all $z \in Z \cap I$. Now since $R$ has characteristic different from 2 , and $d(Z) \subseteq Z$, we find that $d(x) z-x d(z)=0$ for all $x \in I$ and for all $z \in Z \cap I$. Substituting $x y$, where $y \in I$ for $x$ in the last relation and using the same again we conclude that $d(x) y z=0$ for all $x, y \in I$ and for all $z \in Z \cap I$. But since $Z^{*}=Z$ and $I^{*}=I$, we also have $Z^{*} \cap I^{*}=Z \cap I$. These arguments show that $d(x) y z^{*}=0$ for all $x, y \in I$ and for all $z \in Z \cap I$. Finally we infer that $d(x) I z=\{0\}=d(x) I z^{*}$ for all $x \in I$ and for all $z \in Z \cap I$. Lemma 1 and the fact that $Z \cap I \neq\{0\}$ insure that $d(x)=0$ for all $x \in I$. Replacing $x$ by $x r$, where $r \in R$ in the last relation and using the same again we arrive at $I d(r)=\{0\}$. This implies that $s I d(r)=\{0\}=s^{*} I d(r)$, where $0 \neq s \in R$. Finally by Lemma 1, we obtain that $d=0$.

Theorem 8. Let $R$ be a *-prime ring of characteristic different from 2 and $I$ a nonzero $*$-ideal of $R$ such that $Z \cap I \neq\{0\}$. Then there exists no nonzero derivation $d$ which commutes with ' $*$ ' and satisfies either $(i) d(x)$ oy $=d(x)$ od $(y)$ for all $x, y \in$ I or (ii) $\operatorname{xod}(y)=d(x)$ od $(y)$ for all $x, y \in I$.

Proof. (i) By hypothesis we have $d(x) y+y d(x)-d(x) d(y)-d(y) d(x)=0$ for all $x, y \in I$. Let $z \in Z \cap I$. Replacing $x$ by $z$ in the hypothesis, we arrive at $d(z) y+$ $y d(z)-d(z) d(y)-d(y) d(z)=0$ for all $y \in I$ and for all $z \in Z \cap I$. But since $R$ has characteristic different from 2 and $d(Z) \subseteq Z$ we arrive at $d(z) y-d(z) d(y)=0$ for all $y \in I$ and for all $z \in Z \cap I$. Now we infer that $d(z) I(d(y)-y)=\{0\}$ for all $y \in I$ and for all $z \in Z \cap I$. But it is obvious to see that $Z^{*} \cap I^{*}=Z \cap I$. Since $d *=* d$, we also observe that $\{d(z)\}^{*} I(d(y)-y)=\{0\}$ for all $y \in I$ and for all $z \in Z \cap I$. Using Lemma 1 , we obtain that $d(z)=0$ for all $z \in Z \cap I$ or $d(y)=y$ for all $y \in I$. If first case holds, then hypothesis gives us $d(x) o z=0$ for all $x \in I$ and for all $z \in Z \cap I$. Since $R$ has characteristic different from 2, Lemma 7 provides us $d(x) z=0$ for all $x \in I$ and for all $z \in Z \cap I$. This implies that $d(x) I z=\{0\}=d(x) I z^{*}$ for all $x \in I$ and for all $z \in Z \cap I$. Lemma 1 and the fact that $Z \cap I \neq\{0\}$ insure that $d(x)=0$ for all $x \in I$. Now arguing in the similar way as in the above Theorem 7, we conclude that $d=0$. If second case holds, then we have $d(y)=y$ for all $y \in I$. Putting $y r$, where $r \in R$ in the last relation and using the same again we conclude that $y d(r)=0$ for all $y \in I$ and for all $r \in R$. This shows that $l I d(r)=\{0\}=l^{*} I d(r)$ for all $r \in R$ and $0 \neq l \in R$. Finally Lemma 1 
gives our required result.

(i i ) Using similar arguments as above, one can obtain the proof.

The following example justifies that "characteristic different from 2" in the hypothesis of the above Theorems 7 and 8 is not superfluous.

Example 4. Consider $R, D, d, *_{e x}, I$ and $d$ as discussed in the Example 1. It is obvious to observe that $Z \cap d=d \neq\{0\}$. It is easy to check that $(i) D(x) o y=x o D(y)$ for all $x, y \in \mathcal{l}$, (ii) $D(x) o y=D(x) o D(y)$ for all $x, y \in d$ and (iii) $x o D(y)=$ $D(x) o D(y)$ for all $x, y \in \mathcal{d}$. However $D \neq 0$.

Theorem 9. Let $R$ be $a *$-prime ring of characteristic different from 2 and $I$ a nonzero *-ideal of $R$ such that $Z \cap I \neq\{0\}$. If $R$ admits a nonzero derivation $d$ which commutes with ' $*$ ' and satisfies $d(x) \operatorname{od}(y)=x$ oy for all $x, y \in I$, then $R$ is commutative.

Proof. Given that $d(x) o d(y)=x o y$ for all $x, y \in I$. Choose $z \in Z \cap I$. Replacing $y$ by $y z$ in the hypothesis we obtain that $d(x) o d(y z)=x o y z$ for all $x \in I$ and for all $z \in Z \cap I$. Now we have

$$
\begin{aligned}
d(x) o d(y z) & =d(x)(d(y) z+y d(z))+(d(y) z+y d(z)) d(x) \\
& =d(x) d(y) z+d(x) y d(z)+d(y) z d(x)+y d(z) d(x)
\end{aligned}
$$

and on the other hand using the hypothesis we obtain that

$$
\begin{aligned}
\text { xoyz } & =(x \circ y) z \\
& =(d(x) o d(y)) z \\
& =d(x) d(y) z+d(y) d(x) z .
\end{aligned}
$$

Equating the above two expressions and using the fact that $d(Z) \subseteq Z$, we conclude that $(d(x) y+y d(x)) d(z)=0$ i.e.; $(d(x) y+y d(x)) I d(z)=\{0\}$ for all $x, y \in I, z \in$ $Z \cap I$. It is obvious that $Z^{*} \cap I^{*}=Z \cap I$. Since $d *=* d$, we also infer that $(d(x) y+y d(x)) I\{d(z)\}^{*}=\{0\}$ for all $x, y \in I, z \in Z \cap I$. By Lemma 1 we arrive at $(d(x) y+y d(x))=0$ for all $x, y \in I$ or $d(z)=0$ for all $z \in Z \cap I$. We claim that $d(z) \neq 0$ for all $z \in Z \cap I$. For otherwise hypothesis provides us $x o z=2 x z=$ $d(x) \operatorname{od}(z)=0$ for all $x \in I$ and $z \in Z \cap I$. Since $R$ has characteristic different from 2, Lemma 7 insures that $x z=0$ i.e.; $x I z=\{0\}$ for all $x \in I, z \in Z \cap I$. Since $Z^{*} \cap I^{*}=Z \cap I$. This fact shows that $x I z^{*}=\{0\}$ for all $x \in I, z \in Z \cap I$. By Lemma 1, we deduce that either $I=\{0\}$ or $Z \cap I=\{0\}$. This leads to a contradiction. Finally we conclude that $(d(x) y+y d(x))=0$ for all $x, y \in I$. Replacing $y$ by $y r$, where $r \in I$ in the last relation and using the same again we obtain that $y[d(x), r]=0$ for all $x, y \in I, r \in R$. This implies that $l I[d(x), R]=\{0\}=l^{*} I[d(x), R]$, for all $x \in I$, where $0 \neq l \in R$. By Lemma 1 , we find that $d(I) \subseteq Z$. Finally using Lemma 4, we get that $R$ is commutative. 
Theorem 10. Let $R$ be a $*$-prime ring of characteristic different from 2 and $I$ a nonzero $*$-ideal of $R$ such that $Z \cap I \neq\{0\}$. If $R$ admits a nonzero derivation $d$ which commutes with ' $*$ ' and satisfies $(d(x)$ oy $)-(\operatorname{xod}(y)) \in Z$ for all $x, y \in I$, then $R$ is commutative.

Proof. Replacing $y$ by $z$, where $z \in Z \cap I$ in the hypothesis and using the fact that $d(Z) \subseteq Z$, we arrive at $2(d(x) z-x d(z)) \in Z$ for all $x \in I$. This implies that $(d(x) z-x d(z)) \in Z$ for all $x \in I$ and for all $z \in Z \cap I$ and hence $(d(x) z-$ $x d(z)) x=x(d(x) z-x d(z))$ for all $x \in I$ and for all $z \in Z \cap I$. In turn we conclude that $(d(x) x-x d(x)) z=0$ i.e.; $(d(x) x-x d(x)) I z=\{0\}$ for all $x \in I$ and for all $z \in Z \cap I$. Since $Z^{*} \cap I^{*}=Z \cap I$, we obtain that $(d(x) x-x d(x)) I z^{*}=\{0\}$ for all $x \in I$ and for all $z \in Z \cap I$. Now hypothesis and Lemma 1 provide us that $(d(x) x-x d(x))=0$ i.e.; $[d(x), x]=0$ for all $x \in I$. Finally Lemma 3 , completes the proof.

The following example demonstrates that the $*$-primeness in the hypothesis in the above theorem is necessary.

Example 5. Let $S=M_{2 \times 2}(\mathbb{R}[x])$, the ring of all $2 \times 2$ matrices over ring $\mathbb{R}[x]$, where $\mathbb{R}[x]$ is the polynomial ring over ring of real numbers. Suppose that $R=S \times S$, which is clearly a ring of characteristic different from 2. Define $D: R \longrightarrow R$ as $D(A, B)=\left(0, D^{\prime}(B)\right)$, where

$$
D^{\prime}\left(\begin{array}{ll}
f(x) & g(x) \\
h(x) & u(x)
\end{array}\right)=\left(\begin{array}{ll}
d(f(x)) & d(g(x)) \\
d(h(x)) & d(u(x))
\end{array}\right),
$$

$B=\left(\begin{array}{ll}f(x) & g(x) \\ h(x) & u(x)\end{array}\right)$ and $d$ is the usual differentiation of the polynomial ring $\mathbb{R}[x]$. Also define $*: R \longrightarrow R$ as $*(A, B)=\left(A^{t}, B^{t}\right)$, where $A^{t}$ and $B^{t}$ are the transpose of the matrices $A$ and $B$ respectively. It can be easily shown that $D$ and $*$ are a nonzero derivation and an involution of $R$ respectively such that $D *=* D$. Suppose that $I=M_{2 \times 2}(\mathbb{R}[x]) \times\{0\}$. It is obvious that $I$ is a $*$-ideal of $R$ and $Z \cap I \neq\{0\}$. Let $0 \neq$ $U, 0 \neq V \in M_{2 \times 2}(\mathbb{R}[x])$. Then we have $(U, 0) R(0, V)=\{(0,0)\}=(U, 0) R(0, V)^{*}$, where $(0,0) \neq(U, 0),(0,0) \neq(0, V) \in R$. This implies that $R$ is not a $*$-prime ring but it is a semiprime ring. Here it is obvious to see that $(D(p) o q)-(p o D(q)) \in Z$ for all $p, q \in I$, but $R$ is noncommutative.

\section{ACKNOWLEDGEMENT}

The authors are indebted to the referee for his/her useful suggestions and valuable comments.

\section{REFERENCES}

[1] M. Ashraf and N. Rehman, "On commutativity of rings with derivations," Results Math., vol. 42, pp. 3-8, 2003. 
[2] H. E. Bell and M. N. Daif, "On derivations and commutativity in prime rings," Acta Math. Hungar., vol. 66, no. 4, pp. 337-343, 1995.

[3] I. N. Herstein, "A note on derivations," Canad. Math. Bull., vol. 21, pp. 369-370, 1978.

[4] M. Hongon, "A note on semiprime rings with derivations," Int. J. Math. \& Math Sci, vol. 2, no. 2, pp. 413-415, 1997.

[5] L. Oukhtite, "Posner's second theorem for Jordan ideals in rings with involution," Expositiones Mathematicae, vol. 2, pp. 415-419, 2011.

[6] L. Oukhtite and S. Salhi, "Derivations and commutativity of *-prime rings," Int. J. Contemp. Math. Sci., vol. 1, no. 9, pp. 439-448, 2006.

[7] L. Oukhtite and S. Salhi, "Derivations as homomorphisms and antihomomorphisms in $*$-prime rings," Algebras, Groups and Geometries, vol. 2, no. 1, pp. 67-72, 2006.

[8] L. Oukhtite and S. Salhi, "On commutativity of *-prime rings," Glasnik Matematicki, vol. 4, no. 1, pp. 57-64, 2006.

[9] L. Oukhtite and S. Salhi, "On derivations in *-prime rings," Int. J. Algebra, vol. 1, no. 5, pp. 241-246, 2007.

[10] J. Vukman, "A note on Jordan *-derivations in semiprime rings with involution," Int. Math. Forum, vol. 1, no. 13, pp. 617-622, 2006.

[11] J. Vukman and I. Kosi-Ulbl, "On centralizers of semiprime rings with involution," Studia Sci. Math. Hungarica, vol. 43, no. 1, pp. 61-67, 2006.

Authors' addresses

Mohammad Ashraf

Department of Mathematics, Aligarh Muslim University, Aligarh, -202002 (India),

E-mail address: mashraf80@hotmail.com

\section{Mohammad Aslam Siddeeque}

Department of Mathematics, Aligarh Muslim University, Aligarh, -202002 (India),

E-mail address: aslamsiddeeque@gmail.com 